\title{
Paper
}

\section{Implementation of C-PolSK}

Modulation in FSO Network and

Analysis of its Atmospheric Performance

\author{
Diptangshu Chattopadhyay ${ }^{1}$ and Manisha Bharti $^{2}$ \\ ${ }^{1}$ Department of Electronics and Communication Engineering, Jalpaiguri Government Engineering College, Jalpaiguri, India \\ ${ }^{2}$ Department of Electronics and Communication Engineering, National Institute of Technology, Delhi, India
}

\begin{abstract}
Free Space Optical (FSO) communication is a quickly developing technology that is designed to handle high data rates while maintaining a good quality factor (Q-factor). Many novel techniques have been implemented to cope with atmospheric attenuating factors, such as fog and rain. Here, we have introduced circular polarization shift keying (C-PolSK) modulation to produce a stable and uniform light beam. The plane of polarized light keeps rotating along with the propagation vector, which helps reduce phase variations and cross-channel effects. C-PolSK provides higher penetrating power and attenuation resistance over linear polarization shift keying (L-PolSK). Two different C-PolSK models have been proposed and graphical comparisons, relying on such parameters as Q-factor, transmission distance and inputoutput power, have been made. The cases of moderate fog and rain attenuation are taken to verify the model's efficiency. Polarized light is split into two orthogonal components and then modulated using the Mach-Zehnder modulator. One of the models also employs the principle of orthogonal differential phase shifting. Eye diagrams have been provided to verify the bit error rate (BER). The orthogonal differential phase shifted C-PolSK model proves to be the most suitable implementation prototype.
\end{abstract}

Keywords-attenuation, bit error rate, circular polarization shift keying, quality factor.

\section{Introduction}

\subsection{Overview}

Free Space Optical communication is emerging as a potential alternative to traditional optical fiber networks. As there is no requirement for physical links between the transmitter and receiver, it may be successfully implemented in remote areas and in difficult terrain. It is also a cost-efficient system with a low bit error rate and a high quality factor. FSO systems use light as the carrier medium and provide a wide bandwidth for data transfer. Unfortunately, signals become attenuated by atmospheric factors, such as rain, fog and snow [1]. In order to provide a steady connection, vari- ous combinations of modulators, multiplexers and coding formats are being tested. All of them have their own sets of advantages and disadvantages. The main objective of this paper is to introduce circular polarization shift keying in FSO test models and to study their performance based on such parameters as transmission distance, quality factor and input-output power. Since polarization of light is a stable and data efficient phenomenon, it is expected to offer significant improvements in terms of performance.

The traditionally used on-off-keying format lacks these features. C-PolSK is considered better than linear polarized shift keying (L-PolSK), because of its high penetration and attenuation resistance properties [2], [3]. It is so because in C-PolSK, the polarized electric field vector travels along a continuous helical path, instead of a straight planar path. The transmitting and receiving antennas can be placed at any orientation, facing the same direction [4]. Quarter wave plates are used for polarization interchange. Two different designs of the C-PolSK modulation technique, transferring data at a rate of $10 \mathrm{Gbps}$, have been proposed. The simulations are performed for fog and rain attenuation using Optisystem 16.0 software. Conclusions concerning the performance efficiency of the proposed models have been drawn based on a comparative analysis of the parametric graphs. Eye diagrams help were relied upon as well.

\subsection{Linear and Circular Polarization of Light}

Most sources emit light beams in which the electric field vectors vibrate in all planes perpendicular to the direction of propagation. If the vibration of electric field is restricted to a single plane by using special materials called polaroids, the beam becomes a linear polarized light beam. Polarization is a stable characteristic and provides resistance from atmospheric attenuation. It has been observed that a polarized carrier is capable of delivering stable output optical power, which is vital for the peak power restriction system. Two types of linear polarization are distinguished: vertical, where the polarized wave is perpendicular to the ground, 
and horizontal, where the polarized wave is parallel to the ground.

The light, after being polarized, may be split into two mutually orthogonal components, using a polarization splitter. These carrier components are modulated with the same or different messages independently. Hence, the signal sending capacity of the system is doubled. The orthogonal components may be combined or split according to the actual requirements. The receiver must be aligned in the same plane as the transmitter for successful detection of the linear plane polarized signal. Linear polarization of light is shown in Fig. 1.

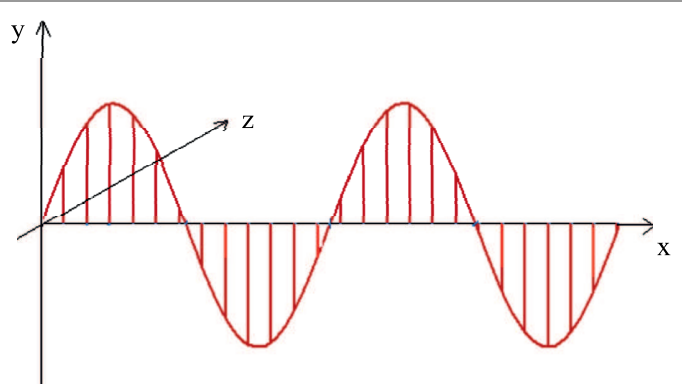

Fig. 1. Linear polarization of light.

Circular polarization of light offers properties that are similar to those of linear polarization of amplitude and frequency. Here the plane of the polarized light rotates continuously with propagation. The direction of rotation may be either clockwise or anti-clockwise. The wave travels in a helical pattern, completing one complete revolution within a wavelength. As the path is changing continuously, it is more resistant to atmospheric turbulence. Penetration power also increases significantly [3]. A circular polarization antenna has the ability to reduce harmful influence due to the multipath phenomenon and renders the signal insensitive to planar orientation at the transmitting and receiving ends [4]. Figure 2 illustrates circular polarization

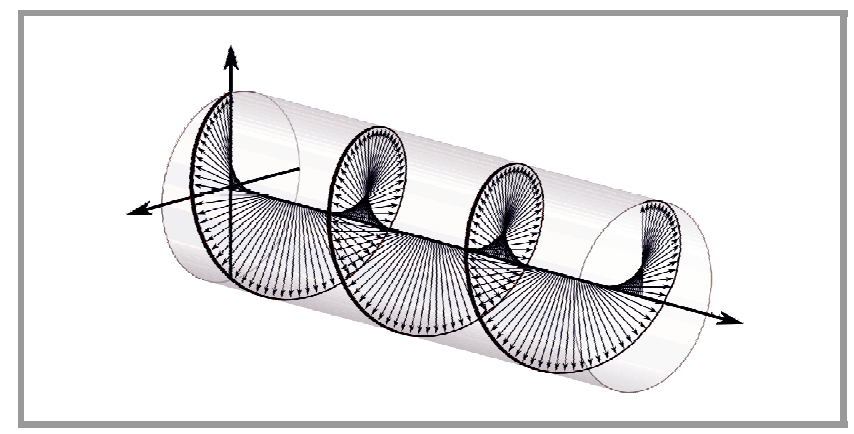

Fig. 2. Circular polarization of light.

of light [5]. Another cause of attenuation affecting electromagnetic waves is the Faraday effect. It consists in phenomenal interaction between light and magnetic fields. The Faraday effect is severe at low frequencies and only affects linear polarized light beams.

\section{FSO System Description and Simulation Parameters}

\subsection{C-PolSK Model 1}

The signal generation process starts with a user defined bit sequence generator producing a string of pre-defined binary numbers at a rate determined by the user. The output is sent to a non-return-to-zero (NRZ) pulse generator which creates a sequence of electric pulses coded by an input digital signal. A continuous laser beam is used as the carrier. The beam is polarized using a polarization controller and is split into two orthogonal components. The device angle is set at 0 degrees for each of the polarizing instruments. One of the split beams is modulated with a message signal from the NRZ pulse generator, using the Mach-Zehnder modulator. The other component is simply passed on to the polarization combiner, where it is combined with the modulated signal to form a single beam again. The beam is then passed through a quarter wave-plate at the angle of $45^{\circ}$ with respect to the device base. A wave-plate works on the principle of birefringence and produces a phase shift between the two orthogonal polarizations of a light wave [6], [7]. The quarter wave-plate having a phase shift of $90^{\circ}$ converts linearly polarized input light into circularly polarized light [8]. Figure 3 illustrates the general working principle of a quarter wave-plate [9].

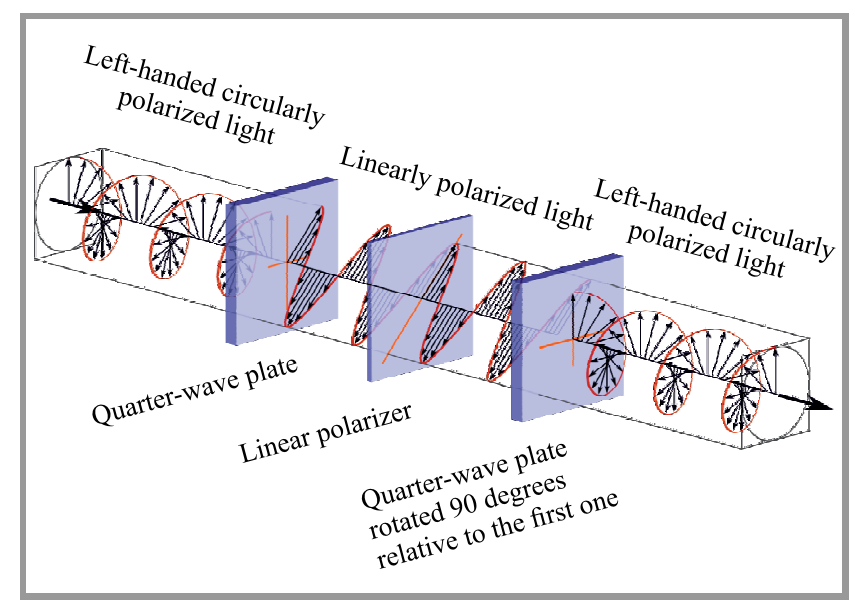

Fig. 3. Operation of a quarter wave-plate.

Link parameters and attenuation values of the FSO channel are set in accordance with the investigatory weather data. After transmission, another quarter wave plate changes the polarization format back to linear. The beams are split once again and the individual components are sent to photodiodes. Here, the optical signals are converted into electrical signals and are then filtered to remove higher frequency components. The BER analyzer provides the eye diagrams and information related to the quality factor and the bit error rate. Figure 4 shows the Optisystem simulation diagram for C-PolSK model 1. 


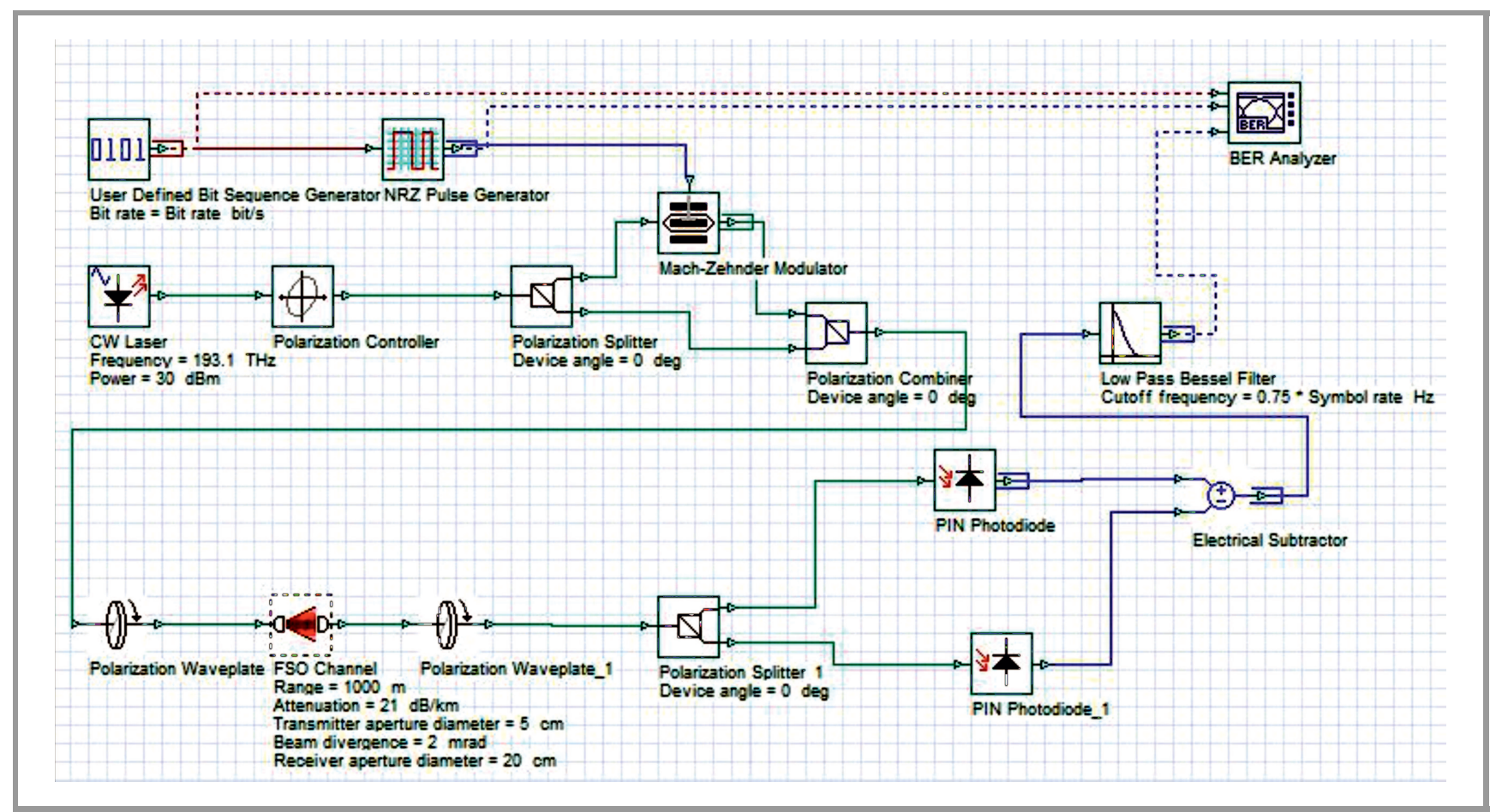

Fig. 4. Simulated FSO network, C-PolSK model 1.

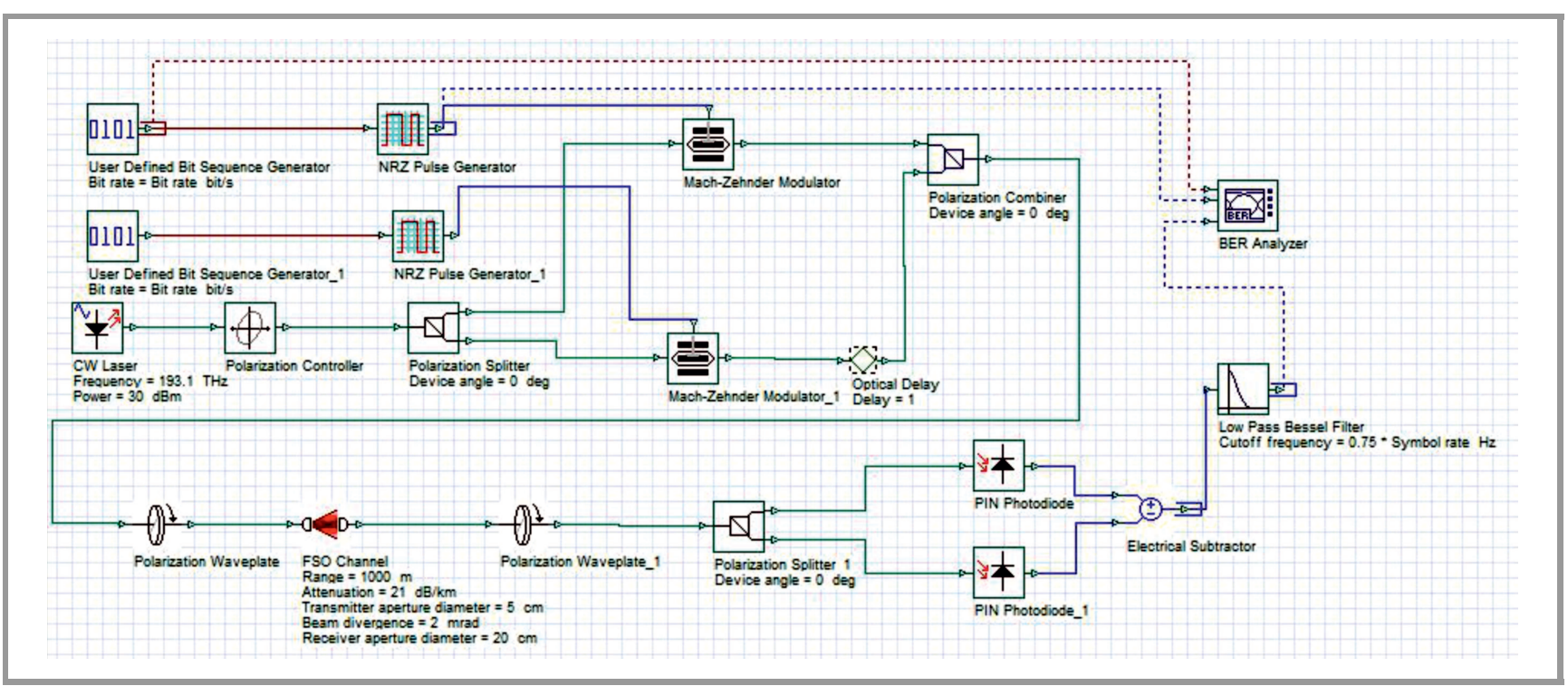

Fig. 5. Simulated FSO network, C-PolSK model 2.

\subsection{PolSK Model 2}

The second model is a slight modification of the first $\mathrm{C}$ PolSK FSO network. Here, we are using the orthogonal differential phase shifting technique along with polarization for data transfer. After being linear polarized, the carrier laser beam is split into two orthogonal components. Both of them are modulated with the same message signal coming from two different sets of user-defined bit sequence generators. One of the modulated beams is passed through an optical delay in which its phase is delayed by a pre-de- fined value. The polarization combiner adds the modulated signal and the phase-delayed modulated signal to produce a differential phase shifted beam [10]. Polarization of the beam is changed to the circular format using a quarter wave plate, as described above, before being transported via an FSO link. At the receiver's end, the same procedures are followed as in model 1 . The differential phase shift demodulation process here is relatively easy, because separate photodiodes are employed to analyze each orthogonal component. There is no need for interferometric devices, such as time and phase delayers. The electrical signal powers 
are subtracted and filtered through a low-pass Bessel filter to remove high frequency components. The result is obtained in a BER analyzer. A network simulation based on this scenario is illustrated in Fig. 5.

\subsection{Simulation Parameters and Values}

Most of the components in the designed FSO network use default values of the Optisystem software simulator. Channel attenuation and laser power inputs are varied accordingly in due course of our investigation. The quarter wave plate device is set at the angle of $+45^{\circ}$ to change the polarization pattern from linear to right circular. Any other angle would produce an elliptical polarized wave instead. The optical delay component shifts the message sequence by 1 bit. The important parameters and their values are listed in Table 1.

Table 1

Simulation parameters and their values

\begin{tabular}{|l|c|}
\hline \multicolumn{1}{|c|}{ Parameters } & Values \\
\hline \hline $\begin{array}{l}\text { Bit rate of user defined bit } \\
\text { sequence generator }\end{array}$ & $10 \mathrm{Gbps}$ \\
\hline Frequency of CW laser & $193.1 \mathrm{THz}$ \\
\hline Transmitter aperture diameter & $5 \mathrm{~cm}$ \\
\hline Beam divergence & $2 \mathrm{mrad}$ \\
\hline Receiver aperture diameter & $20 \mathrm{~cm}$ \\
\hline Receiver responsiveness & $1 \mathrm{~A} / \mathrm{W}$ \\
\hline
\end{tabular}

\section{Impact of Atmospheric Attenuation}

Atmospheric attenuation may be described in terms of absorption and scattering coefficients. While absorption decreases signal strength, scattering redistributes the energy in arbitrary directions. Q-factor is reduced and BER increases significantly. We have considered rain and fog attenuations here, categorizing them based on their intensity levels. According to the empirical model for Mie scattering, specific attenuation caused by fog is given as:

$$
\beta_{f o g}=\frac{3.91}{V}\left(\frac{\lambda}{550}\right)^{-q}
$$

where $\lambda$ is the wavelength of the propagating beam in nanometers, $V$ is visibility in kilometers, and $q$ is the size distribution of particles [9].

The Kim model defines $q$ as:

$$
\begin{array}{ll}
0 & \text { for } \quad V<0.5 \mathrm{~km} . \\
V-0.5 & \text { for } \quad 0.5 \mathrm{~km}<V<1 \mathrm{~km} . \\
0.16 V+0.34 & \text { for } \quad 1 \mathrm{~km}<V<6 \mathrm{~km} . \\
1.3 & \text { for } \quad 6 \mathrm{~km}<V<50 \mathrm{~km} . \\
1.6 & \text { for } \quad V>50 \mathrm{~km} .
\end{array}
$$

Based on visibility categories, three different types of fog have been enlisted and a sample value from each category is taken as reference. The wavelength of the light beam equals $1550 \mathrm{~nm}$ and attenuation is calculated for each specific case. The data are presented in Table 2.

Table 2

Types of fog and their specific attenuation

\begin{tabular}{|c|c|c|}
\hline $\begin{array}{c}\text { Visibility } \\
{[\mathrm{km}]}\end{array}$ & $\begin{array}{c}\text { Specific attenuation } \\
{[\mathrm{dB} / \mathrm{km}]}\end{array}$ & Type \\
\hline \hline 1 & 9 & Light fog \\
\hline 0.5 & 21 & Medium fog \\
\hline 0.2 & 34 & Heavy fog \\
\hline
\end{tabular}

Specific size rain droplets are also responsible for signal scattering. Specific attenuation increases exponentially with the rainfall rate. Both of them are related by the empirical formula given below [11]:

$$
\beta_{\text {rain }}=1.076 R^{0.67} \text {. }
$$

Rainfall too has been categorized into three different groups on the basis of precipitation rate. Going by the values calculated from Eq. (2), the following Table 3 has been prepared depicting the relationship between rainfall type, precipitation rate and specific attenuation [12].

Table 3

Types of rain and their specific attenuation

\begin{tabular}{|c|c|c|}
\hline Rainfall type & $\begin{array}{c}\text { Rainfall rate } \\
{[\mathrm{mm} / \mathrm{hr}]}\end{array}$ & $\begin{array}{c}\text { Specific attenuation } \\
{[\mathrm{dB} / \mathrm{km}]}\end{array}$ \\
\hline \hline Light rain & 0.25 & 0.425 \\
\hline Medium rain & 12.5 & 5.88 \\
\hline Heavy rain & 25 & 9.25 \\
\hline
\end{tabular}

The values in Tables 2 and 3 are taken as standard values in our entire investigatory work. Other attenuation factors, such as beam loss, free space loss, pointing loss and scintillation, have been ignored.

\section{Results and Discussions}

Q-factor is a concise measure of the quality of signal which takes into consideration the effects of cross-talks, noise and distortions. Q-factor vs. transmission distance graphs have been plotted for both C-PolSK models. The cases of moderate fog attenuation and moderate rain attenuations have been taken. Network performance in other atmospheric situations follows the same graphical trend.

From Figs. 6 and 7, we can see that the Q-factor decreases with the increase in transmission distance. It is more apparent in the case of fog attenuation. The second model that is based on the combined principle of differential phase shifting and circular polarization shifting, provides a much better result than the simple circular polarized model (model 1). 


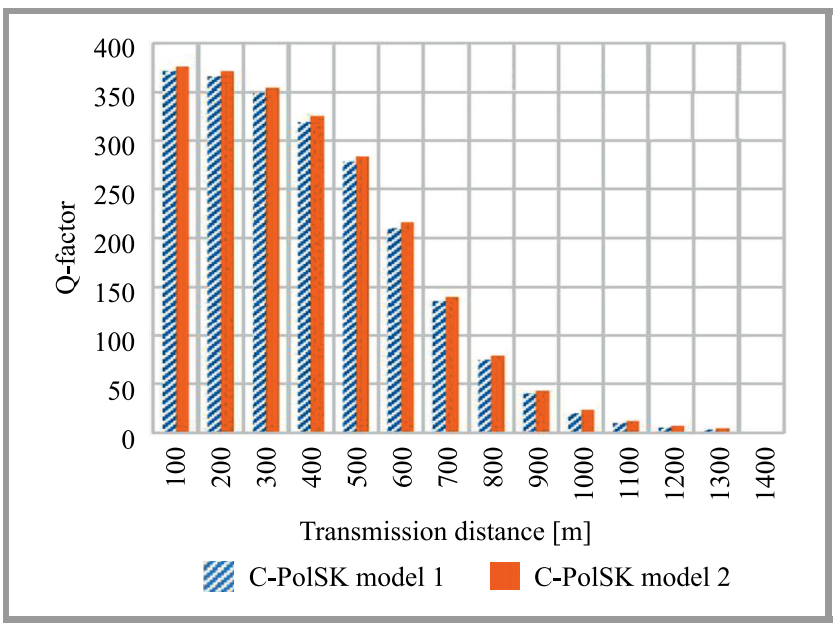

Fig. 6. Q-factor vs. transmission distance in moderate fog.

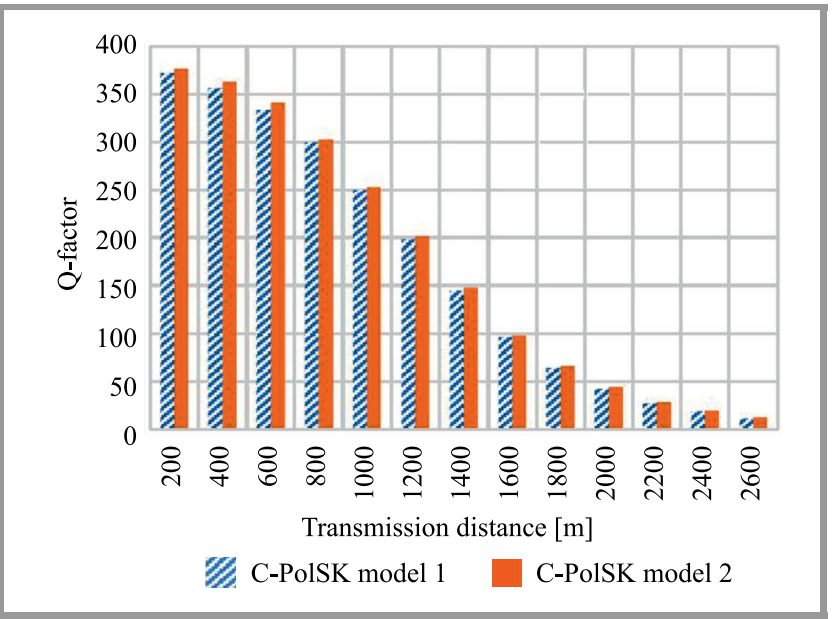

Fig. 7. Q-factor vs. transmission distance in moderate rain.

The amount of power consumed by the system is an important parameter in determining its overall performance. It is also a key economic index. Due to health-related impact, there are some restrictions affecting the power rating of laser beams used in communication networks.

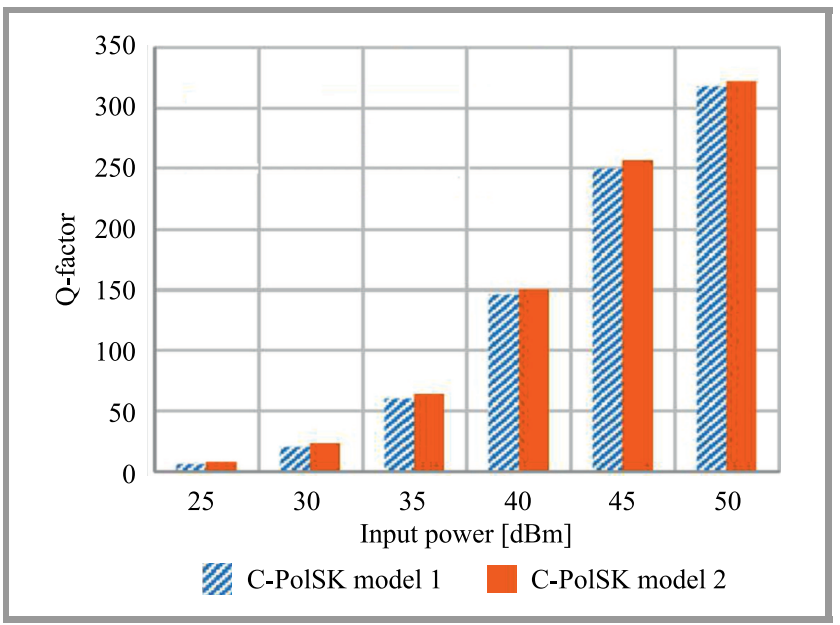

Fig. 8. Q-factor vs. input power in moderate fog.

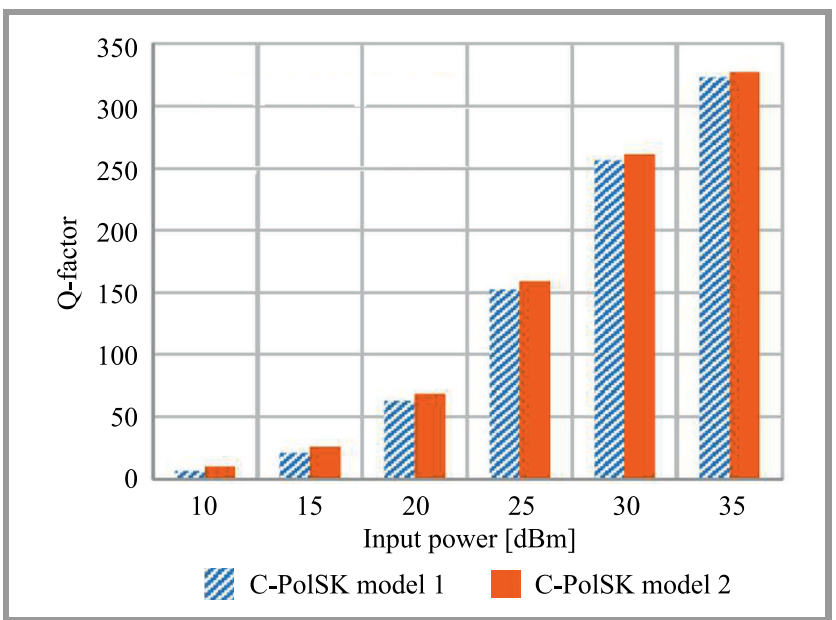

Fig. 9. Q-factor vs. input power in moderate rain.

So, a device has to be efficient while maintaining the required signal quality and keeping the BER within a certain limit. Figures 8 and 9 depict the relationship between the Q-factor of the signal at the receiving end and the input power.

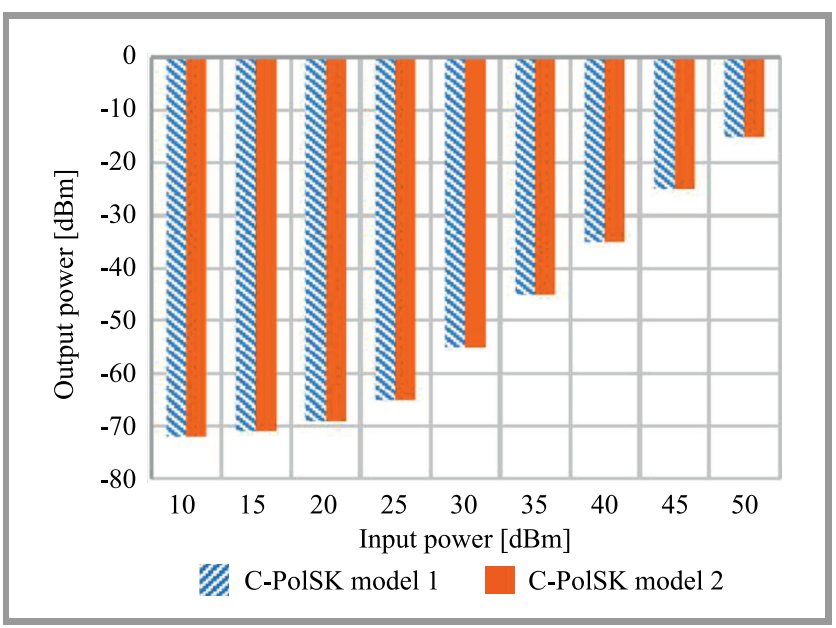

Fig. 10. Output power vs. input power in moderate fog.

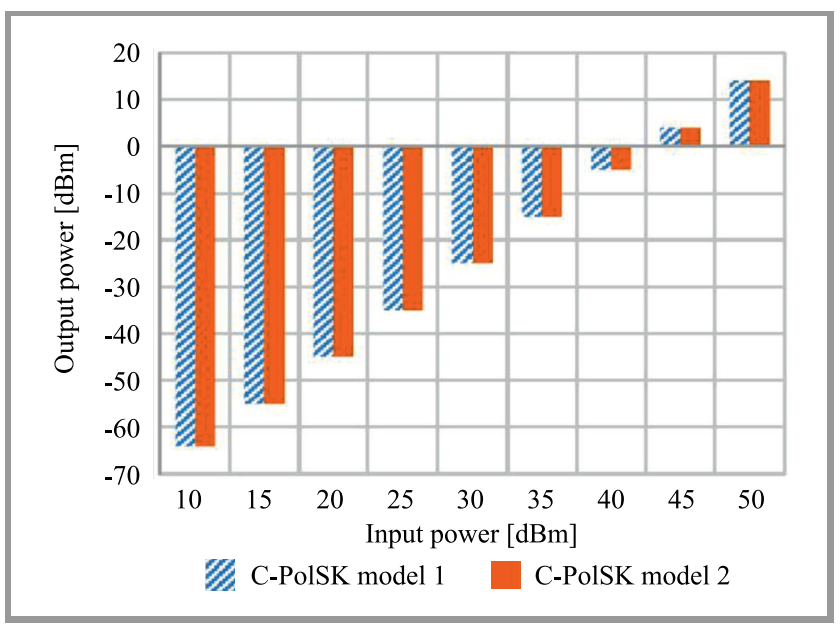

Fig. 11. Output power vs. input power in moderate rain. 
The double modulated C-PolSK model (model 2) utilizes the principle of orthogonal differential phase shifting and produces better results than the simple C-PolSK model (model 1). Since it uses a non-coherent method of detection, there is no need for the demodulator to keep track of a reference wave. Phase shifting provides resistance against atmospheric noise because the amplitude remains constant. So, model 2 proves to be a more reliable way of transferring data. Amplifiers may be used at the transmission and receiving ends to enhance performance.

The objective of the next step would be to compare the power efficiency of both models. Efficiency is defined as the ratio of the output power and the input power. Since the same laser wave is used in both models and a similar methodology is followed, the output power should be nearly the same. No amplifiers have been used in either of the models and the values here are recorded in $\mathrm{dBm}$ (Figs. 10-11).

Power efficiency is the same for both C-PolSK models. At the same output power, the second model provides a better quality factor than model 1. Q-factor and BER are interrelated. A high Q-factor is an indication that the signal has not been distorted too much. The number of error bits is relatively low, and so is BER. Consumption of power in-

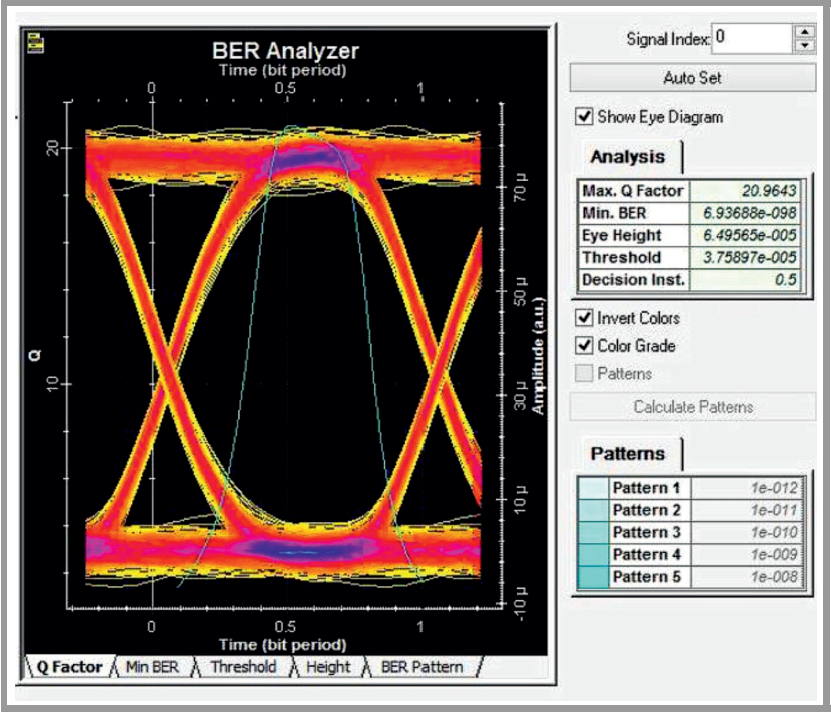

Fig. 12. Eye diagram of C-PolSK model 1 in moderate rain.

creases drastically in foggy weather. Figures 12-13 show the eye diagrams for C-PolSK model 1 and model 2, respectively, recorded in fog attenuated conditions at a distance of $1 \mathrm{~km}$. Minimum BER, maximum quality factor, eye height, threshold and decision instant values are visible as well.

\section{Conclusion}

After investigating the parametric results, one may conclude that the results of the second C-PolSK model are much better than those of the first model. Low BER, high efficiency

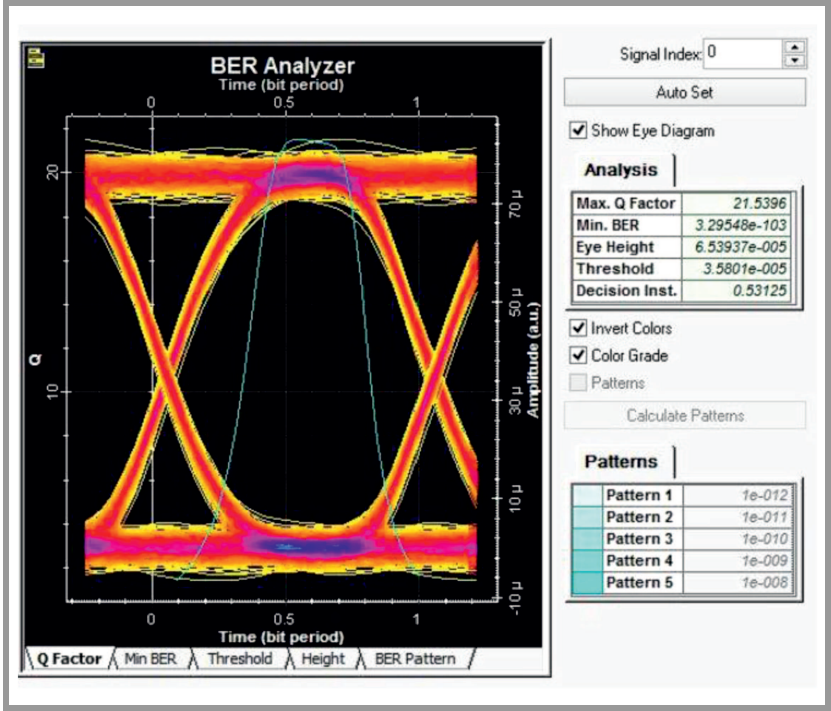

Fig. 13. Eye diagram of C-PolSK model 2 in moderate rain.

and a good quality factor justify our conclusion. It is a form of orthogonal differential phase shifting. But the demodulation process is easier because the orthogonal components are analyzed by separate photodiodes. In the case of circular polarization, the plane of the polarized light keeps on rotating, creating a helical path. The data rate may be enhanced by multiplexing and using multi beams for propagation. Due to all of their features, C-PolSK models may be used in inter-satellite communication. The signal may travel at much longer distances due to low turbulence and attenuation. Further development of the technology would exert a pragmatic effect on the performance of upcoming FSO networks.

\section{References}

[1] A. Malik and P. Singh, "Free space optics: current applications and future challenges", Int. J. of Optics, vol. 2015, Article ID 945483 , 2015 (doi: 10.1155/2015/945483).

[2] P. Wang and Z. Liu, "The analysis of methods and performance of atmospheric laser communication based on polarization shift keying modulation (PolSK) technology", in Proc. of Symp. on Photonics and Optoelec., Shanghai, China, 2012 (doi: 10.1109./SOPO.2012.6270451).

[3] J. T. Boyd, W. E. Kock, and S. K. Yang, "Circular polarization at light wavelengths", Proceedings of the IEEE, vol. 65, no. 89, pp. 1218-1219, 1977 (doi: 10.1109/PROC.1977.10681).

[4] S. Sharma and C. C. Tripathi, "A comprehensive study on circularly polarized antenna", in Proc. 2nd Int. Innov. Appl. of Comput. Intell. on Power, Energy and Controls with Their Impact on Humanity CIPECH 2016, Ghaziabad, India, 2016, pp. 234-239 (doi: 10.1109/CIPECH.2016.7918773).

[5] "Circular polarization" [Online]. Available: https://en.wikipedia.org/wiki/Circular_polarization

[6] G. Zhang and Y. Wang, "Study of Jones matrix of the birefringent crystal", in Proc. of Symp. on Photonics and Optoelec., Wuhan, China, 2009 (doi: 10.1109/SOPO.2009.5230257).

[7] W. Wang, "Determining the retardation of a wave plate by using spectroscopic method", Optics Commun., vol. 285, no. 24, 2012, pp. 4850-4855 (doi: 10.1016/j.optcom.2012.08.011). 
[8] P. S. Theocaris and E. E. Gdoutos, "Passage of polarized light through optical elements", in Matrix Theory of Photoelasticity. Springer Series in Optical Sciences, vol. 11, pp. 45-81. Berlin, Heidelberg: Springer, 1979 (ISBN: 9783540357896).

[9] "Waveplate" [Online]. Available: https://en.wikipedia.org/ wiki/Waveplate

[10] H. Q. Guo, G. F. Chen, and T. Y. Zhang, "Circle polarization phase shift keying technology research based on space laser communication system", in Proc. of the Int. Conf. on Comp. Inform. Syst. and Indust. Appl. CISIA 2015, Bangkok, Thailand, 2015 (doi: 10.2991/cisia-15.2015.43).

[11] H. Kaushal, V. K. Jain, and S. Kar, Free Space Optical Communication. Springer (India), 2017 (978-81-322-3887-4).

[12] Z. Ghassemlooy and W. O. Popoola, "Terrestrial free-space optical communications", in Mobile and Wireless Communications Network Layer and Circuit Level Design, S. A. Fares and F. Adachi, Eds. InTech, 2010 (ISBN: 9789533070421).

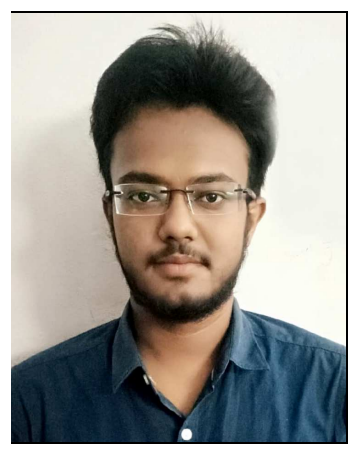

Diptangshu Chattopadhyay is currently pursuing his Bachelor of Technology degree in Electronics and Communication Engineering from Jalpaiguri Government Engineering College, West Bengal. He was a summer intern at the National Institute of Technology, Delhi, in 2019, where he worked extensively on FSO communication networks. His research areas include wireless communication networks and signal processing.
https://orcid.org/0000-0002-8788-873X

Email: diptangshu2010@gmail.com

Department of Electronics and Communication

Engineering

Jalpaiguri Government Engineering College

Danguajhar

Jalpaiguri 735102, India

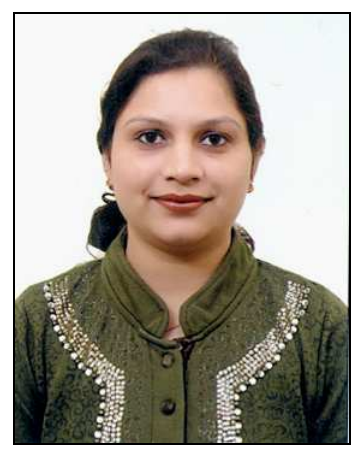

Manisha Bharti received her Ph.D. in Optical Communication and Networks from IKG Punjab Technical University, Jalandhar, in 2017. She is currently working as an Assistant Professor at the National Institute of Technology (NIT), Delhi, and has over 16 years of experience in academics. Her areas of research include optical communication and networks, analog, digital and broadband communication circuits, optical signal processing and VLSI.

Email: manishabharti@nitdelhi.ac.in

Department of Electronics and Communication

Engineering

National Institute of Technology

Delhi, Sector A-7

Institutional Area, Narela

Delhi - 110040, India 